\title{
Thirty year trend (1967-1996) in prevalence of poliomyelitis and vaccine coverage in Ballabgarh, Haryana, India
}

\author{
K Anand, S Kant, G Kumar, S K Kapoor
}

India is committed to eradication of poliomyelitis by the year 2000. ${ }^{1}$ However, India still reported about $58 \%$ of all polio cases documented globally in $1995 .^{2}$ India has adopted the World Health Organisation's recommended strategy of National Immunization Days (that is, Pulse Polio Immunization) from the year 1995. Oral polio vaccine (OPV) was introduced at national level in $1985 .{ }^{1}$ However, no community based surveillance has been instituted. The data from the sentinel centres based in cities suggest a decline in the incidence of poliomyelitis. ${ }^{34}$ As the surveillance is passive and the extent of under reporting is not known, sentinel surveillance may not reflect the true situation in the community.

We report our experience with oral polio immunisation and its impact on prevalence of poliomyelitis in Ballabgarh Block of Haryana state in Northern India. The information on poliomyelitis occurrence in this community is available for past 30 years.

\section{Methods}

Comprehensive Rural Health Services Project (CRHSP) at Ballabgarh, located in the state of Haryana, is $35 \mathrm{~km}$ away from Delhi. The project area consists of approximately 70000 people residing in 28 villages. OPV was introduced in this area in 1980 when it was given together with the triple antigen (DPT) vaccine in the field at domiciliary level. In 1985 , it was decided to give OPV to all eligible subjects on a fixed day in a month. On the day of immunisation, the required amount of vaccine is supplied to the health post located in the village. This practice not only reduced the wastage of OPV but also ensured better maintenance of cold chain. Since December 1995, pulse polio immunisation of all under five year old children on two days in a year has been introduced in this area as at national level.

Active surveillance is carried out by the health workers who routinely visit each house once in 15 days. During this visit, they provide domiciliary services like immunisation, antenatal care, etc, and enquire about any morbidity. All children under 15 years of age with lameness with no other obvious cause (trauma) and a compatible history (history of fever followed by paralysis of one or more limbs) were classified as acute flaccid paralysis (AFP). All cases of AFP are then seen by a doctor for confirmation of diagnosis.

As per the government of India guidelines, all cases of AFP are to be notified to the district immunisation officer. On receipt of this notifi- cation, a health team led by district immunisation officer visits the case and collects stool sample. The stool samples are sent to the National Institute of Communicable Diseases (NICD), which is the apex body for virological surveillance for poliomyelitis in the country. This process of virological confirmation of AFP cases was started in the study area in 1996. This process has been strengthened during the last six months following the government of India's adoption of global initiative on polio eradication. Since then, virological confirmation has been sought for all the cases of AFP that have come to our notice.

The records were maintained manually until 1987 when they were computerised. Once a month, on a specified date, workers of one subcentre come to the headquarter of the project at Ballabgarh. During this visit they update the database by entering any new information like immunisations, deliveries, deaths, morbidity, etc, gathered during the previous month. The database is in Dbase package. Among the various fields for each person, the fields relevant for this study are that of age, sex, morbidity, and uniqueno. Uniqueno refers to a number given to each person residing in the study area. This 12 digit alphanumeric number is based on Primary Health centre (2), subcentre (1), village (1), house number (4), family number (2), individual's number (2) in the family. The coding of morbidly has been developed in house and all cases of post-polio residual paralysis are coded as " 4 ".

Information on poliomyelitis is further supplemented by a chronic disease morbidity, which is carried out as a part of the annual census. Every year, during the months of May and June, the health workers make house to house visits to update the family demographic data. All new information collected during this census including chronic morbidity is fed into the computerised database. Thus, even if a case of AFP was missed during the domiciliary visit of the worker, this case would be identified during the annual survey. For the estimation of prevalence, the computerised database was used.

For the period 1967-81 when there was no active AFP surveillance, the prevalence has been taken from a study conducted in this area. ${ }^{5}$ This study was conducted in the same 28 villages of CRHSP to estimate the prevalence of common neurological diseases. Briefly, the methodology involved two steps. The first step was screening by an interview schedule administered by the multipurpose workers. The posi- 
Table 1 Prevalence of poliomyelitis in children under 5 years old during the period 1967-1996 in Ballabgarh, Haryana, India

\begin{tabular}{|c|c|c|c|c|c|c|}
\hline Source & Reference date & $\begin{array}{l}\text { Age group } \\
\text { (y) }\end{array}$ & Birth cohort & $\begin{array}{l}\text { Children } \\
\text { surveyed }\end{array}$ & $\begin{array}{l}\text { Children with } \\
\text { lameness }\end{array}$ & $\begin{array}{l}\text { Prevalence per } \\
1000\end{array}$ \\
\hline \multirow[t]{2}{*}{ Prevalence survey (Kapoor et al) } & December 1981 & $5-14$ & $1967-76$ & 13773 & 156 & 11.3 \\
\hline & & $0-5$ & $1977-81$ & 8575 & 99 & 11.5 \\
\hline \multirow[t]{3}{*}{ Computerised database } & December 1996 & $10-14$ & $1982-86$ & 8490 & 41 & 4.8 \\
\hline & & $5-10$ & $1987-91$ & 9552 & 33 & 3.4 \\
\hline & & $0-5$ & $1992-96$ & 10422 & 5 & 0.5 \\
\hline
\end{tabular}

tive cases were then confirmed by examination by a doctor. The sensitivity and reliability of the multipurpose workers in identifying those with paralysis of any part of the body using an interview schedule was also tested. Both were found to be $100 \%$. Subsequently on clinical examination by a doctor, $70 \%$ of paralysis cases were attributed to poliomyelitis

The annual vaccine coverage rates with three doses of OPV among 12-23 months old children was estimated from the computerised data base. These estimates have been confirmed by independent surveys conducted by undergraduate and postgraduate medical students. Children were classified, based on their age, into five year birth cohorts, except for the period 1967-76. This was a 10 year cohort as the break up was not available in five year groups. The prevalence of poliomyelitis was plotted on a log scale and the vaccine coverage on a linear scale.

\section{Results}

The coverage with OPV showed a steady increase since its introduction to reach $90 \%$ by 1990 (fig 1). Since 1990, coverage with three doses of OPV has consistently been more than $90 \%$ in the project area.

The prevalence of poliomyelitis in the pre-immunisation period (before 1978) was around 11.5 per 1000 among under five children. The decline in prevalence started in the early 1980 s when the immunisation coverage reached $40 \%$. A more pronounced decline has been noticed since 1990 when the coverage had reached above $90 \%$. At present the prevalence is 0.5 per thousand under five children. In the past few years, four cases of AFP had been reported from our area. One of these was subsequently confirmed to be caused by poliomyelitis based on stool culture examination.

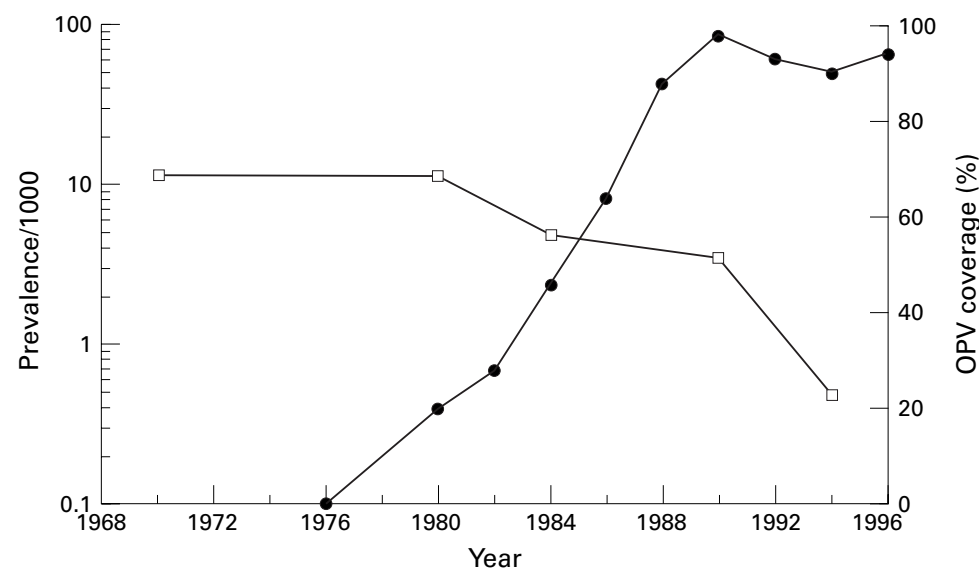

Figure 1 Polio prevalence (0-5 years) and OPV coverage (12-23 months) in Ballabgarh, India.

\section{Discussion}

This paper shows the trend in the prevalence of poliomyelitis with increasing vaccine coverage from a community based data. Previous studies on trend of poliomyelitis have either been sentinel centre based ${ }^{3}$ or for a short period of time. ${ }^{67}$

The case definition of poliomyelitis used in this study is based only on clinical criteria. This may overestimate the prevalence of poliomyelitis as non-poliomyelitic causes of lameness would also be included in this definition. In the absence of any specific intervention, the number of lameness cases that result from non-poliomyelitis causes is likely to remain at the same level or show a more gradual decline over the entire study period compared with poliomyelitis induced lameness. This would mean that the overestimation is likely to be proportionately more in the later years. Therefore, the decline in the prevalence of poliomyelitis is probably more than what is being reflected in figure 1 .

This study used data on prevalence of lameness from two sources including one from a published study. The observed decline in polio prevalence between 1967-1981 and 19821996 could have been because of methodological differences between the two studies. However, the villages covered and the multipurpose workers involved were the same and one of the investigator of the earlier study (SKK) was involved subsequently in the data collection for rest of the period. Therefore, we believe that the methodology of both the studies were essentially similar. Thus, combining the two sources of data seems valid.

The strengths of our study are that (1) The sensitivity and reliability of the workers involved in the study was assessed and found to be $100 \%$. Therefore, the diagnosis of cases appears to be valid. (2) The community was kept constantly under active surveillance for AFP for the past 15 years. The completeness of the surveillance was confirmed by an independent survey for chronic diseases including lameness by the trainee medical interns, which was conducted in a part of the study area during the year 1996-97. This survey did not identify any new case of lameness that were not represented in the database. (3) The data cover a period of 30 years with estimates of prevalence of lameness at five different points in time. This allows us to draw valid inference about the trend of lameness in the community. (4) The accurate coverage rates are available on annual basis since the introduction of OPV in the community. (5) This study allows us to assess the impact on prevalence of poliomyelitis when the coverage rates are maintained at 
more than $90 \%$ level for a reasonable period (more than five years).

The prevalence of poliomyelitis had declined to 0.5 per 1000 for children under five after five years of high coverage. This is equivalent to one new case occurring every year in the 10411 children of the study area. It is difficult to say whether the declining trend would have continued until the elimination of polio by routine immunisation alone.

Pulse polio immunisation was introduced in 1995 in this area and the coverage during these campaigns have been more than $98 \%$. Our data provide a baseline to assess the impact of pulse polio immunisation.
1 Basu RN. Poliomyelitis in India. Indian Pediatr 1991;28:589-92.

2 World Health Organization. Expanded programme on immunization: progress towards poliomyelitis eradication. Weekly Epidemiological Record 1996;71:53-4.

3 Sharma M, Sen M, Ahuja B, et al. Paralytic poliomyelitis 1976-1988: report from a sentinel centre. Indian Pediatr 1990;27:143-50.

4 Mandke BV, Pawar RM, Naik DD, et al. AM. Review of poliomyelitis in Bombay (1982-1989): observations on the improved immunization coverage. Indian 7 Public Health 1990;34:200-8.

5 Kapoor SK, Banerjee AK. Prevalence of common neurological diseases in a rural community of India. Indian $\mathcal{F}$ Community Medicine 1989;14:171-5.

6 Khajuria R, Datta N, Kumar R, et al. Impact of annual immunization programme with oral polio vaccine on the prevalence of paralytic poliomyelitis. Indian $\mathcal{\text { f Pediatr } 1 9 8 9 ;}$ prevalence

7 Srinivasa DK, Sahai A, Rotti SB, et al. Poliomyelitis trends in Pondicherry, South India, 1989-91. f Epidemiol Community Health 1997;51:443-8. 